\title{
Use of postmenopausal hormone therapy and risk of Alzheimer's disease in Finland: nationwide case-control study
}

\author{
Hanna Savolainen-Peltonen, ${ }^{1,2}$ Päivi Rahkola-Soisalo, ${ }^{1}$ Fabian Hoti, ${ }^{3}$ Pia Vattulainen, ${ }^{3}$ \\ Mika Gissler, ${ }^{4,5,6}$ Olavi Ylikorkala, ${ }^{1}$ Tomi S Mikkola ${ }^{1,2}$
}

Check for updates

University of Helsinki and

Helsinki University Hospital,

Obstetrics and Gynecology,

Haartmaninkatu 2, PO Box

140, FIN-00029 HUS, 00029

Helsinki, Finland

${ }^{2}$ Folkhälsan Research Center, Biomedicum, Helsinki, Finland

${ }^{3}$ EPID Research Oy, Espoo,

Finland

${ }^{4}$ National Institute for Health and Welfare, Helsinki, Finland

${ }^{5}$ Karolinska Institute,

Department of Neurobiology,

Care Sciences and Society,

Division of Family Medicine,

Huddinge, Sweden

${ }^{6}$ University of Turku, Research

Centre for Child Psychiatry,

Turku, Finland

Correspondence to: TS Mikkola tomi.mikkola@hus.fi

(ORCID 0000-0003-2049-088X) Additional material is published online only. To view please visit the journal online.

Cite this as: $B M J$ 2019;364:1665 http://dx.doi.org/10.1136/bmj.1665

Accepted: 1 February 2019

\section{ABSTRACT}

OBJECTIVES

To compare the use of hormone therapy between Finnish postmenopausal women with and without a diagnosis for Alzheimer's disease.

DESIGN

Nationwide case-control study.

SETTING

Finnish national population and drug register, between 1999 and 2013.

\section{PARTICIPANTS}

All postmenopausal women $(n=84739)$ in Finland who, between 1999 and 2013, received a diagnosis of Alzheimer's disease from a neurologist or geriatrician, and who were identified from a national drug register. Control women without a diagnosis $(n=84739)$, matched by age and hospital district, were traced from the Finnish national population register.

INTERVENTIONS

Data on hormone therapy use were obtained from the Finnish national drug reimbursement register.

\section{MAIN OUTCOME MEASURES}

Odds ratios and $95 \%$ confidence intervals for Alzheimer's disease, calculated with conditional logistic regression analysis.

RESULTS

In 83688 (98.8\%) women, a diagnosis for Alzheimer's disease was made at the age of 60 years or older, and 47239 (55.7\%) women had been over 80 years of age at diagnosis. Use of systemic hormone therapy was associated with a 9-17\% increased risk of Alzheimer's disease. The risk of the disease did not differ significantly between users of estradiol only (odds

\section{WHAT IS ALREADY KNOWN ON THIS TOPIC}

Data on the association between use of postmenopausal hormone therapy and risk of Alzheimer's disease are conflicting

Several observational studies have indicated that hormone therapy might have a protective effect on the risk of Alzheimer's disease, but this was not supported by the placebo controlled Women's Health Initiative Memory Study

These findings were later challenged by the timing hypothesis, which indicates that oestrogen could be neuroprotective only if it is started soon after the onset of menopause

\section{WHAT THIS STUDY ADDS}

Use of postmenopausal systemic hormone therapy is accompanied with an increase in the risk of Alzheimer's disease in postmenopausal women, whereas the use of vaginal estradiol shows no such risk

Particularly long term exposure to hormone therapy is associated with an increased risk of Alzheimer's disease, but the increase in risk is not dependent on the age at treatment initiation ratio $1.09,95 \%$ confidence interval 1.05 to 1.14$)$ and those of oestrogen-progestogen $(1.17,1.13$ to 1.21$)$. The risk increases in users of oestrogen-progestogen therapy were not related to different progestogens (noreth isterone acetate, medroxyprogesterone acetate, or other progestogens); but in women younger than 60 at hormone therapy initiation, these risk increases were associated with hormone therapy exposure over 10 years. Furthermore, the age at initiation of systemic hormone therapy was not a decisive determinant for the increase in risk of Alzheimer's disease. The exclusive use of vaginal estradiol did not affect the risk of the disease $(0.99$, 0.96 to 1.01$)$.

\section{CONCLUSIONS}

Long term use of systemic hormone therapy might be accompanied with an overall increased risk of Alzheimer's disease, which is not related to the type of progestogen or the age at initiation of systemic hormone therapy. By contrast, use of vaginal estradiol shows no such risk. Even though the absolute risk increase for Alzheimer's disease is small, our data should be implemented into information for present and future users of hormone therapy.

\section{Introduction}

Alzheimer's disease, the most common cause of dementia, occurs more frequently in women than in men. ${ }^{1}$ This difference might be due to the longer life expectancy of women, but sex specific differences in the incidence of Alzheimer's disease might also exist. ${ }^{1-3}$ It is known that oestrogens exert neuroprotection in several animal studies. ${ }^{4-6}$ Also, oestrogen deficiency as a result of early menopause has been associated with an increased risk of Alzheimer's disease. ${ }^{7}$ Therefore, prolonging the oestrogen supply with postmenopausal hormone therapy could protect against Alzheimer's disease.

However, clinical data on the association between hormone therapy and the disease have remained inconclusive. Despite several observational studies supporting the protective effect of hormone therapy on Alzheimer's disease, ${ }^{8-13}$ a subsequent placebo controlled trial (the Women's Health Initiative Memory Study (WHIMS)) failed to confirm this benefit, and in fact implied an increased risk of overall dementia in hormone therapy users. ${ }^{14} 15$ The conflicting data could in part result from differences in the study design, study populations, or hormone therapy regimens. Unlike clinical practice, hormone therapy in the WHIMS trial was initiated in women aged 65 or older. ${ }^{1415}$ Thus, one explanation might also be the timing hypothesis, which suggests that oestrogen 
could be neuroprotective only if started soon after the onset of menopause. ${ }^{16}$ This hypothesis originates from cardiovascular studies ${ }^{17}$ where the age at the start of hormone therapy appears to predict the cardiovascular effects of hormone therapy. Treatment initiated before age 60 is protective, but if started at a later age, it is detrimental towards the vasculature. Such a window for hormone therapy use has also been suggested for cognitive effects. ${ }^{16}$

By using Finnish comprehensive nationwide registers, we were able to conduct a case-control comparison to investigate whether hormone therapy had an effect on the risk of Alzheimer's disease, and whether this risk was associated with age of treatment initiation or duration of treatment use.

\section{Methods}

In Finland, patients with Alzheimer's disease are entitled to $40 \%$ reimbursement for treatment from national health insurance, but this requires a statement from a neurologist or geriatrician. They must base the diagnosis on symptoms consistent of mild or moderate Alzheimer's disease, decrease in social capacity for at least three months, cognitive tests, magnetic resonance imaging or computed tomography scanning of the brain, and exclusion of alternative diagnoses. The physician also must confirm whether the patient has other dementia related diseases, such as Lewy body dementia or mixed dementia. For mixed dementia, patients are entitled to reimbursement only if the symptoms and findings are caused mainly by Alzheimer's disease. In total, 84739 women with a diagnosis for Alzheimer's disease were entered into this register in 1999-2013. During the same period of time, control women without a diagnosis were identified from the Finnish National population register (one control per case; $n=84739$ ). Control women were matched with cases by age (within 1 month) and by hospital district according to the woman's municipality of residence. Hospital districts were further divided into five university hospital districts.

Finland has a reliable nationwide register that includes all hormone therapy users from year 1994. Use of the treatment in Finland is available only with a physician's prescription, and regimens are partly $(40-60 \%)$ reimbursed by the government. At each pharmacy visit, hormone therapy purchases are entered into the drug reimbursement register, confirming use of the prescribed regimen of hormone therapy. The register was initiated in 1994, so we could not differentiate whether a woman who bought the treatment in 1994 was a new user or was continuing her treatment initiated before 1994. Therefore, we assumed that all systemic users older than 52 years in 1994 had initiated the treatment at age 52 and vaginal users at age 65 , which are the mean ages at systemic and vaginal initiation of hormone therapy in our study population, respectively. ${ }^{18}$ This approximation has been used in several previous studies. ${ }^{19-21}$ However, we also analysed separately women who had started hormone therapy in 1995 (one year after the register opened) or later (that is, fresh starters: 65102 cases and 65102 controls), because this group's detailed history of treatment use was documented in the register. The findings in this subanalysis were fully in line with those in the whole study population, so the data of this subanalysis are not shown.

The regimens of systemic hormone therapy in Finland contain exclusively estradiol, which is given either orally $(90 \%)$ or transdermally $(10 \%)$. The regimens identified by trade names were transformed into doses of estradiol (oral or transdermal). Various progestogens were used in combination with estradiol (that is, oestrogen-progestogen therapy), of which norethisterone acetate and medroxyprogesterone acetate were the most common. ${ }^{20}$ According to the Finnish guidelines, only women who have had hysterectomies can use estradiol without progestogen, and these women were studied as an estradiol only group. Oral estradiol doses in Finland are usually 1-2 $\mathrm{mg} /$ day, and transdermal (gel or patch) estradiol is used with equivalent doses (25-100 $\mu \mathrm{g} /$ day). However, owing to the switching of the use of hormone therapy from one route to another and to the relatively similar route independent levels of circulating oestrogen, we did no subanalyses according to the treatment route. Sequential users of oestrogen-progestogen therapy were defined as women who used estradiol with 1014 days of progestogen courses each month, or at intervals of one to three months. Women who used both estradiol and progestogen every day were considered as continuous users of oestrogen-progestogen therapy. Tibolone users were considered as a separate group. Users of vaginal estradiol only (Vagifem, NovoNordisk, Copenhagen, Denmark; $25 \mu \mathrm{g}$ twice a week) were analysed separately.

Exposure to hormone therapy (ever use) was considered to have started from the date of the first purchase, or from age 52 if systemic hormone therapy was used at the register opening or from age 65 years if vaginal estradiol was used at register opening. Cumulative exposure to hormone therapy was classified by duration (lasting $\leq 3$ years, $>3$ to $\leq 5$ years, $>5$ to $\leq 10$ years, or $>10$ years). We assessed the time period from treatment initiation to diagnosis for Alzheimer's disease. To address the potential critical time window for oestrogen brain effect, ${ }^{16}$ we also compared the risk of the disease in women who had started hormone therapy aged under 60 versus those aged 60 and over.

\section{Statistical methods}

We used a conditional logistic regression analysis to estimate, by using odds ratios with $95 \%$ confidence intervals, the relative risk of Alzheimer's disease associated with different regimens of hormone therapy. The association between age at treatment initiation and Alzheimer's disease was also analysed with conditional logistic regression, using both continuous age and five years age classes as variables. We used the $\chi^{2}$ test to test differences between categorical variables. Differences between continuous variables 
were evaluated with the student's $t$ test or two tailed test of equal or given proportions. Significance was set at $\mathrm{P}<0.05$.

\section{Patient and public involvement}

No patients were involved in the setting of the research question or the outcome measures, nor were they involved in the developing plans for design or implementation of the study. No patients were asked to advise on interpretation or writing up of the results. There are no plans to disseminate the results of the research to study participants or the relevant patient community.

\section{Results}

Overall, in 83688 (98.8\%) women, a diagnosis for Alzheimer's disease was made at the age of 60 years or older, and 47239 (55.7\%) women had been over 80 years of age at diagnosis (table 1). Time from initiation of hormone therapy to diagnosis was shorter than five years in $886(5.6 \%)$ women, but longer than 10 years in 11805 (74.9\%). At the time of diagnosis, only 2305 (14.6\%) women were still using hormone therapy, whereas 10188 (64.6\%) had stopped treatment more than three years before diagnosis.

Patients with Alzheimer's disease used systemic hormone therapy more often $(18.6 \% \quad v \quad 17.0 \%$, respectively, $\mathrm{P}<0.001$ ) but used vaginal estradiol only less often $(12.7 \% v 13.2 \%, \mathrm{P}=0.005)$ than controls (table 1). The mean exposure time to systemic hormone therapy did not differ between patients with Alzheimer's disease and control women (table 1). The relative proportions of different progestogens users were similar in cases and controls (table 1), but exposure to oestrogen-progestogen therapy with other or mixed progestogens was longer in patients with Alzheimer's disease $(\mathrm{P}=0.02)$.

Overall, hormone therapy users with Alzheimer's disease $(n=11456,73.7 \%)$ had started systemic hormone therapy under age 60 years less often than controls $(n=10662,75.1 \%, P=0.006)$. The mean age at initiation of estradiol only was similar for patients with Alzheimer's disease and controls (table 1), but the mean age at initiation of oestrogen-progestogen therapy was slightly higher for patients with Alzheimer's disease than for controls (56.1 (standard deviation 7.8) v 55.8 (7.6), $\mathrm{P}=0.02)$.

\begin{tabular}{|c|c|c|c|}
\hline & Patients with Alzheimer's disease $(n=84739)$ & Controls $(n=84739)$ & $\mathbf{P}$ \\
\hline \multicolumn{4}{|c|}{ Start of follow-up (age at Alzheimer diagnosis (years) for cases) } \\
\hline$<50$ & $99(0.1)$ & $101(0.1)$ & - \\
\hline $50-54$ & $273(0.3)$ & $274(0.3)$ & - \\
\hline $55-59$ & $679(0.8)$ & $678(0.8)$ & - \\
\hline $60-64$ & $1373(1.6)$ & $1373(1.6)$ & - \\
\hline 65-69 & $4028(4.8)$ & $4028(4.7)$ & - \\
\hline $70-74$ & $10731(12.7)$ & $10785(12.7)$ & - \\
\hline 75-79 & $20317(24.0)$ & $20311(24.0)$ & - \\
\hline$\geq 80$ & $47239(55.7)$ & $47193(55.7)$ & - \\
\hline \multicolumn{4}{|c|}{ University healthcare district at the time of diagnosis } \\
\hline Northern (Oulu) & $14794(17.5)$ & $14746(17.4)$ & - \\
\hline Eastern (Kuopio) & $17425(20.6)$ & $17450(20.6)$ & - \\
\hline Southern (Helsinki) & $24053(28.4)$ & $23956(28.3)$ & - \\
\hline Western (Turku) & $10856(12.8)$ & $10873(12.8)$ & - \\
\hline Central Finland (Tampere) & $17574(20.7)$ & $17624(20.8)$ & - \\
\hline Unknown & $6(0.01)$ & $3(0.0)$ & - \\
\hline Abroad & $31(0.04)$ & $87(0.1)$ & - \\
\hline \multicolumn{4}{|l|}{ Hormone therapy use } \\
\hline No use & $58186(68.7)$ & $59175(69.8)$ & \multirow[t]{2}{*}{$<0.001$} \\
\hline Systemic use & $15768(18.6)$ & $14394(17.0)$ & \\
\hline Estradiol only & $5606(35.6)$ & $5312(36.9)$ & \multirow[t]{2}{*}{0.01} \\
\hline EPT & $9941(63.0)$ & $8890(61.9)$ & \\
\hline EPT with MPA & $1955(19.7)$ & $1795(20.1)$ & \multirow[t]{3}{*}{0.27} \\
\hline EPT with NETA & $3080(31.0)$ & $2661(29.8)$ & \\
\hline EPT with other* or mixed progestogens & $4906(49.4)$ & $4434(50.0)$ & \\
\hline Tibolone & $221(1.4)$ & $192(1.3)$ & 0.17 \\
\hline Vaginal estradiol & $10785(12.7)$ & $11170(13.2)$ & 0.005 \\
\hline \multicolumn{4}{|c|}{ Age at hormone therapy initiation (mean, SD) } \\
\hline Estradiol only & $58.0(9.3)$ & $57.7(9.0)$ & 0.1 \\
\hline EPT & $56.1(7.8)$ & $55.8(7.6)$ & 0.02 \\
\hline \multicolumn{4}{|l|}{ Exposure time (mean, SD) } \\
\hline Systemic use & $11.4(8.6)$ & $11.5(8.6)$ & 0.19 \\
\hline Estradiol only & $11.8(9.3)$ & $11.9(9.4)$ & 0.40 \\
\hline EPT & $9.8(7.7)$ & $9.7(7.7)$ & 0.28 \\
\hline EPT with MPA & $9.6(7.9)$ & $9.7(7.7)$ & 0.10 \\
\hline EPT with NETA & $7.0(7.4)$ & $6.7(7.3)$ & 0.28 \\
\hline EPT with other* or mixed progestogens & $11.6(7.4)$ & $11.3(7.4)$ & 0.02 \\
\hline Tibolone & $1.6(1.8)$ & $1.6(1.7)$ & 0.93 \\
\hline
\end{tabular}




\begin{tabular}{|c|c|c|c|c|}
\hline Age at initiation and type of hormone therapy & Patients with Alzheimer's disease (No) & Controls (No) & Odds ratio $(95 \% \mathrm{Cl})$ & $\mathbf{P}$ \\
\hline \multicolumn{5}{|l|}{ Age $<60$ years } \\
\hline No hormone therapy & 48331 & 48925 & 1.00 & - \\
\hline Estradiol only & 3125 & 3042 & $1.06(1.01$ to 1.12$)$ & 0.03 \\
\hline EPT & 6330 & 5812 & 1.14 (1.09 to 1.19$)$ & $<0.005$ \\
\hline EPT with MPA & 1296 & 1247 & $1.08(1.00$ to 1.17$)$ & 0.06 \\
\hline EPT with NETA & 1419 & 1270 & 1.17 (1.08 to 1.26$)$ & $<0.005$ \\
\hline EPT with other* or mixed progestogens & 3615 & 3295 & $1.15(1.09$ to 1.21$)$ & $<0.005$ \\
\hline Tibolone & 83 & 90 & $0.97(0.72$ to 1.32$)$ & 0.86 \\
\hline \multicolumn{5}{|l|}{ Age $\geq 60$ years } \\
\hline No hormone therapy & 45180 & 45635 & 1.00 & - \\
\hline Estradiol only & 1310 & 1157 & 1.15 (1.06 to 1.25$)$ & $<0.005$ \\
\hline EPT & 1630 & 1352 & $1.23(1.14$ to 1.32$)$ & $<0.005$ \\
\hline EPT with MPA & 269 & 227 & $1.21(1.01$ to 1.44$)$ & 0.04 \\
\hline EPT with NETA & 963 & 792 & $1.23(1.12$ to 1.36$)$ & $<0.005$ \\
\hline EPT with other* or mixed progestogens & 398 & 333 & $1.21(1.05$ to 1.41$)$ & 0.009 \\
\hline Tibolone & 90 & 66 & $1.38(1.00$ to 1.89$)$ & 0.05 \\
\hline
\end{tabular}

EPT=oestrogen-progestogen therapy; $N E T A=$ norethisterone acetate; $M P A=$ medroxyprogesterone acetate.

*Other progestogens include levonorgestrel, progesterone, megestrol acetate, lynestrenol, drospirenone, and trimegestone.

In the whole study population, the risk of Alzheimer's disease was increased but did not differ significantly between users of estradiol only (odds ratio 1.09, 95\% confidence interval 1.05 to 1.14 ) and those of oestrogenprogestogen $(1.17,1.13$ to 1.21). Exclusive use of vaginal estradiol was not related to risk of Alzheimer's disease $(0.99,0.96$ to 1.01$)$. In women younger than 60 at hormone therapy initiation, use of the different oestrogen-progestogen therapies was associated with an $8-17 \%$ increased risk of Alzheimer's disease (table 2). We saw no significant differences between the different therapies with regards to Alzheimer's disease risk. Use of estradiol only was associated with a small but significant increase in disease risk. The risk of
Alzheimer's disease in estradiol only users (odds ratio 1.06, 95\% confidence interval 1.01 to 1.12 ) and all users of oestrogen-progestogen therapy $(1.14,1.09$ to 1.19) did not differ significantly. The use of tibolone carried no significant risk of Alzheimer's disease in this age group. The risk increases in users of estradiol only and oestrogen-progestogen therapy were related to 10 years of exposure or more, whereas shorter use of hormone therapy was not associated with Alzheimer's disease risk (table 3).

In women aged 60 or older at the initiation of hormone therapy, use of estradiol only, oestrogenprogestogen therapy, or tibolone was associated with similar increases in risk (15-38\%) for Alzheimer's

Table 3 | Odds ratios for Alzheimer's disease in women younger than 60 or aged 60 and over at treatment initiation of estradiol only or various combined therapies, stratified by duration of treatment

\begin{tabular}{|c|c|c|c|c|}
\hline Type and duration of hormone therapy & Patients with Alzheimer's disease (No) & Controls (No) & Odds ratio $(95 \% \mathrm{Cl})$ & $P$ \\
\hline \multicolumn{5}{|l|}{ Age $<60$ years at treatment initiation } \\
\hline \multicolumn{5}{|l|}{ Estradiol only } \\
\hline No hormone therapy & 44879 & 44978 & 1.00 & - \\
\hline$<3$ years & 126 & 140 & 0.89 (0.69 to 1.15$)$ & 0.38 \\
\hline 3 to $<5$ years & 78 & 60 & $1.31(0.93$ to 1.87$)$ & 0.13 \\
\hline 5 to $<10$ years & 254 & 286 & 0.88 (0.74 to 1.06$)$ & 0.18 \\
\hline$\geq 10$ years & 1989 & 1862 & 1.07 (1.00 to 1.15$)$ & 0.04 \\
\hline \multicolumn{5}{|l|}{ Oestrogen-progestogen therapy } \\
\hline No hormone therapy & 46301 & 46796 & 1.00 & - \\
\hline$<3$ years & 845 & 854 & $1.02(0.92$ to 1.13$)$ & 0.67 \\
\hline 3 to $<5$ years & 401 & 416 & $1.00(0.86$ to 1.15$)$ & 0.98 \\
\hline 5 to $<10$ years & 1118 & 1063 & $1.10(1.00$ to 1.20$)$ & 0.05 \\
\hline$\geq 10$ years & 3355 & 2891 & $1.20(1.13$ to 1.26$)$ & $<0.005$ \\
\hline \multicolumn{5}{|l|}{ Age $\geq 60$ years at treatment initiation } \\
\hline \multicolumn{5}{|l|}{ Estradiol only } \\
\hline No hormone therapy & 43894 & 44062 & 1.00 & - \\
\hline$<3$ years & 905 & 804 & $1.13(1.03$ to 1.25$)$ & 0.01 \\
\hline 3 to $<5$ years & 160 & 119 & 1.35 (1.07 to 1.72$)$ & 0.01 \\
\hline 5 to $<10$ years & 164 & 139 & $1.19(0.95$ to 1.50$)$ & 0.13 \\
\hline$\geq 10$ years & 28 & 27 & 1.04 (0.61 to 1.77 ) & 0.88 \\
\hline \multicolumn{5}{|l|}{ Oestrogen-progestogen therapy } \\
\hline No hormone therapy & 44135 & 44422 & 1.00 & - \\
\hline$<3$ years & 1204 & 1015 & $1.20(1.10$ to 1.30$)$ & $<0.005$ \\
\hline 3 to $<5$ years & 233 & 179 & $1.32(1.08$ to 1.60$)$ & 0.006 \\
\hline 5 to $<10$ years & 198 & 147 & $1.36(1.10$ to 1.69$)$ & $<0.005$ \\
\hline$\geq 10$ years & 17 & 24 & 0.73 (0.39 to 1.35$)$ & 0.31 \\
\hline
\end{tabular}


disease (table 2). In this age group, the increased risk was already detectable after three to five years' use of estradiol only or oestrogen-progestogen therapy (table 3). The increases in risk of Alzheimer's disease between women younger than 60 and those aged 60 or older at treatment initiation did not differ significantly. When the effect of age at treatment initiation on risk of Alzheimer's disease was analysed further in five year intervals, no association emerged (fig 1). Furthermore, age as a continuous variable was no determinant for disease risk in users of estradiol only (odds ratio 1.0, 95\% confidence interval 0.98 to 1.02), oestrogen-progestogen therapy (1.0, 0.99 to 1.01$)$, or any hormone therapy (1.0, 0.99 to 1.01). The disease appeared earlier in women with a history of systemic use of hormone therapy than in those with vaginal estradiol use or without any prior use of the treatment (fig 2).

\section{Discussion \\ Principal findings}

In our large case-control study of 84739 patients with Alzheimer's disease and a similar number of control women, we showed that the systemic use of estradiol only or oestrogen-progestogen therapy was associated with an increased risk of Alzheimer's disease, whereas use of vaginal estradiol showed no such risk. The age at initiation of hormone therapy did not appear to be a determinant for risk of the disease, whereas in women younger than 60 at treatment initiation, the risk increases were associated with exposure to hormone therapy for over 10 years.

\section{Comparison with other studies}

Most observational studies have reported a reduced risk of Alzheimer's disease and all cause dementia in users of hormone therapy. ${ }^{8-1322}$ These analyses can be criticised, owing to the lack of a placebo arm and the possible bias of healthy woman in hormone therapy users. This criticism gained strong support from the placebo controlled WHIMS trial, reporting an increased risk of impaired cognition and probable dementia in women who used conjugated equine oestrogens (CEE)

\begin{tabular}{|c|c|c|}
\hline & $\begin{array}{l}\text { No of cases } \\
\text { /controls }\end{array}$ & $\begin{array}{l}\text { Odds ratio } \\
(95 \% \mathrm{Cl})\end{array}$ \\
\hline \multicolumn{3}{|c|}{$\begin{array}{l}\text { Age at } \\
\text { initiation } \\
\text { of any HT }\end{array}$} \\
\hline $75-79$ & $47 / 60$ & $\longrightarrow$ \\
\hline $70-74$ & $134 / 130$ & \\
\hline $65-69$ & $260 / 277$ & $\longrightarrow$ \\
\hline $60-64$ & $343 / 305$ & \\
\hline $55-59$ & $358 / 365$ & \\
\hline \multirow[t]{2}{*}{$<50-54$} & $3570 / 3579$ & 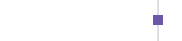 \\
\hline & & 0.5 \\
\hline
\end{tabular}

Fig 1 | Risk of Alzheimer's disease in women initiating systemic hormone therapy (HT) at different ages, as odds ratios and $95 \%$ confidence intervals. Line at 1.0 denotes the risk in the group of women who started systemic use younger than 55 . Numbers of cases and controls are given for five years periods with and without medroxyprogesterone acetate. ${ }^{14} 15$ On the other hand, this study was also criticised because, unlike in normal clinical practice, hormone therapy was initiated for women older than 65, many years after the onset of menopause. Moreover, the WHIMS study did not differentiate Alzheimer's disease from other dementia or cognitive decline, although the pathophysiology of dementia includes multiple different neurodegenerative and vascular processes, which could respond differently to oestrogen exposure.

Our epidemiological study, although large in size and conducted in a case-control setting, can show only associations between hormone therapy use and the risk of Alzheimer's disease. Moreover, the small risk increases are vulnerable to bias from unsuspected sources, which are unavoidable in all observational studies. $^{8-13}$ But if there is a causal relation, it seems that estradiol could be primarily responsible for the increased risk of Alzheimer's disease, because use of estradiol only was related to the increased risk. However, progestogen could potentiate the effect of estradiol on the risk of Alzheimer's disease, because the risk elevations tended to be higher in users of oestrogenprogestogen therapy than users of estradiol only. This notion accords with the WHIMS study finding ${ }^{14} 15$ that the risk of all cause dementia was higher in users of CEE and medroxyprogesterone acetate than in those using CEE only. Progestogens, such as medroxyprogesterone acetate or norethisterone acetate, differ in structure and in their capacity to bind to progesterone and other steroid hormone receptors ${ }^{23}$; therefore, the risk of Alzheimer's disease in users of the different oestrogenprogestogen therapies could vary. Our data show that norethisterone acetate, medroxyprogesterone acetate, or other progestogens as components of oestrogen-progestogen therapy do not differ in regard to risk of Alzheimer's disease. Tibolone, a synthetic steroid hormone with oestrogenic, progestogenic, and androgenic actions, has shown a neuroprotective effect in preclinical studies. ${ }^{24}$ Our finding implies that tibolone does not differentiate significantly from other forms of systemic hormone therapy with regard to the risk of Alzheimer's disease, but owing to the small number of tibolone users, this conclusion must be interpreted with caution.

The cause of Alzheimer's disease is not yet fully understood, but $\beta$ amyloid plaques and neurofibrillary tangles containing hyperphosphorylated $\tau$ protein do accumulate in the brain. ${ }^{25}{ }^{26}$ Several risk factors, such as genetic tendency, head trauma, smoking, and low education could speed up the development of the disease. Multiple full term pregnancies might also predispose to the development of Alzheimer's disease, perhaps due to repeat oestrogen and progesterone surges. ${ }^{27}$ Our present data imply that the prolongation of estradiol exposure beyond the natural menopausal age with the use of hormone therapy could stimulate the progression of Alzheimer's disease, and thus increase its risk, especially if such exposure to estradiol is continued for over 10 years. Our data do not allow any detailed speculations for the possible biological 


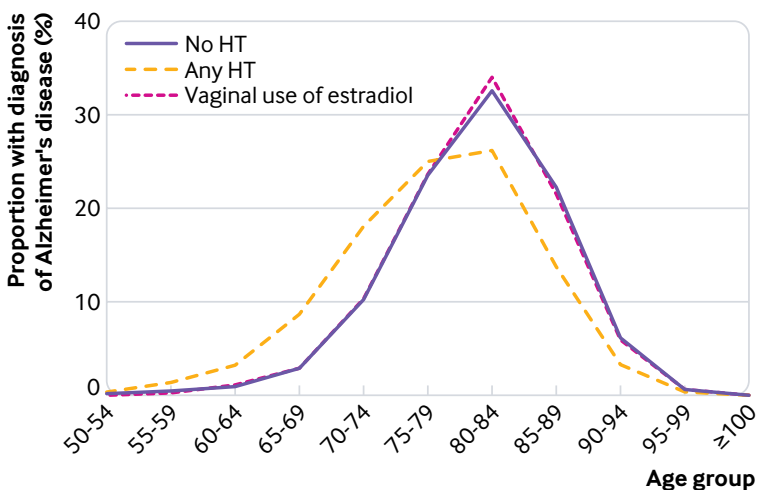

Fig 2 | Proportion (\%) of women with a diagnosis of Alzheimer's disease in different age groups according to systemic use of hormone therapy, vaginal use of estradiol, or without any history of hormone therapy (HT) use

mechanism through which estradiol could have caused this effect. Although estradiol might inhibit the accumulation of $\beta$ amyloid in animal models and in early postmenopausal women, ${ }^{3628}$ it has been speculated that such an effect of oestrogen is lost with advancing age. We sought thoroughly for such a timing effect from our data, but we must conclude that the age at initiation of hormone therapy is no decisive determinant for the risk of Alzheimer's disease in the future.

We have previously shown that the systemic use of estradiol based hormone therapy was associated with a marked difference in the death risk of dementia; the risk of death for vascular dementia was profoundly decreased (by 37-39\%), whereas risk of death for Alzheimer's disease showed a much smaller decrease or even lacked a decrease. ${ }^{21}$ Furthermore, in the extended follow-up of the Women's Health Initiative study, ${ }^{29}$ the mortality for Alzheimer's disease and other dementia was reduced in CEE users, although the authors conclude that this finding should be interpreted cautiously because of multiple comparisons. It is possible that the use of hormone therapy is accompanied, directly or indirectly, with a decreased risk of the most severe forms of Alzheimer's disease, perhaps by improving cardiovascular health. Yet, it is the incidence of and not mortality from Alzheimer's disease that determines the total burden of the disease.

\section{Strengths and limitations of the study}

Our study had several limitations. Firstly, we did not have baseline demographic data for known risk factors for Alzheimer's disease, either for the hormone therapy users or controls. We would not expect this omission to cause many errors in our study, because several common dementia risk factors (hypertension, hypercholesterolaemia, and smoking) were evenly distributed between the two groups in another study from the same population. ${ }^{30}$ Moreover, cardiovascular mortality was reduced in the Finnish hormone therapy users, ${ }^{18} 20$ implying that higher cardiovascular morbidity could not explain the elevated disease risk of hormone therapy users in our study. The ApoE
E4 allele frequency in Finland (at nearly 20\%) is among the highest in the world, ${ }^{31}{ }^{32}$ and some studies suggest that the effects of postmenopausal hormone therapy on Alzheimer's disease are modified by the ApoE status. ${ }^{2}$ ApoE E4 is not screened in the Finnish healthcare system, but it is unlikely that ApoE status would have differed between the two study groups. Furthermore, Finnish users of hormone therapy did not differ in socioeconomic status or education from the non-users, ${ }^{33}$ and healthcare services in Finland are available for everyone, free of charge, or substantially subsidised.

Secondly, due to the first signs of cognitive impairment seven to eight years before the final diagnosis, ${ }^{34}$ there is a possibility that such women sought help from hormone therapy, and thus an increased risk of Alzheimer's disease was a cause for its use and not its consequence. However, this seems unlikely in our population, because the disease diagnosed most often at over age 80 would have caused symptoms at around age $72-73^{34}$; however, most hormone therapy users (74\%) started use under age 60 . Furthermore, only $14 \%$ of users were still on hormone therapy at the time of diagnosis. Thirdly, the duration of the pre-register use of hormone therapy had to be estimated in a quarter of the total study population. However, based on the previous data, ${ }^{20} 21$ such an estimation for hormone therapy use is accurate. Fourthly, controls were not screened with magnetic resonance imaging of the brain or neurological examination to exclude Alzheimer's disease, and thus some controls could have had undiagnosed disease. This chance should not cause any concern because the prevalence of Alzheimer's disease in Europe is $4.4 \%{ }^{35}$ Finally, although we could reliably differentiate between systemic and vaginal use of estradiol, we were not able to compare the use of oral and transdermal preparations with use of cyclic and continuous oestrogen-progestogen therapy.

Our study also had several strengths. Firstly, this study is one of the largest on the association between hormone therapy and Alzheimer's disease. Secondly, it is vital that in a study on women with cognitive decline, the use of hormone therapy was objectively assessed from a reliable nationwide register controlled by authorities, because self reporting is not reliable in patients with poor cognitive performance. Thirdly, Alzheimer's disease was accurately diagnosed and differentiated from vascular dementia and other forms of dementia or cognitive decline according to internationally approved criteria. ${ }^{36}$ Finally, it has been shown that $97 \%$ of Finnish patients with Alzheimer's disease actually use treatment ${ }^{37}$ and thus have been included into the reimbursement register, which confirms that we could reliably detect patients with Alzheimer's disease in Finland.

\section{Conclusions and policy implications}

The present study indicates that the use of systemic hormone therapy, once claimed to be protective against 
Alzheimer's disease, is accompanied with a $9-17 \%$ increase in the risk of the disease in postmenopausal women, whereas the exclusive use of vaginal estradiol shows no risk. In absolute terms, we estimate that nine to 18 excess diagnoses of the disease per year will be detected in 10000 women aged 70-80 (incidence 105 per 10000 women years in this age group), especially in those who had used hormone therapy for over 10 years. The age at initiation of hormone therapy is no decisive determinant for the future risk of Alzheimer's disease. Hormone therapy users should be informed of a possible risk of the disease with prolonged use, even though the absolute risk elevations are small.

Contributors: TSM and OY designed the study. FH, PV, and MG analysed the data. HS-P drafted the figure and the tables. HS-P, PR-S, OY, and TSM drafted the manuscript, and all the authors read, revised the manuscript, and approved its final version. TSM and HS-P are responsible for the overall content as guarantors, and accept full responsibility for the work and the conduct of the study, had access to the data, and controlled the decision to publish. The corresponding author attests that all listed authors meet authorship criteria and that no others meeting the criteria have been omitted, had full access to all the data in the study, and had final responsibility for the decision to submit for publication.

Funding: This study was supported by a Helsinki University Hospital research grant and the Jane and Aatos Erkko Foundation. The funders had no role in the study design; in the collection, analysis, and interpretation of data; in the writing of the report; and in the decision to submit the article for publication. We confirm that the researchers were independent from funders and that all authors had full access to all of the data (including statistical reports and tables) in the study and can take responsibility for the integrity of the data and the accuracy of the data analysis.

Competing interests: All authors have completed the ICMJE uniform disclosure form at www.icmje.org/coi_disclosure.pdf and declare: support from Helsinki University Hospital and the Jane and Aatos Erkko Foundation for the submitted work; speaker and consulting fees for Mylan (HS-P, TSM) and Astellas (TSM); funding for congress trips from Merck, Sharp, and Dohme (HS-P), Astellas (PR-S), and Olympus (PR-S); FH and PV work for EPID Research, which performs financially supported studies for several pharmaceutical companies; no other relationships or activities that could appear to have influenced the submitted work.

Ethical approval: The research committee of the Helsinki University Hospital approved our study plan. Approval from the ethics committee was not needed in our register based study because we did not contact any study patients or their care givers. Appropriate approvals for the use of confidential register data in scientific research were obtained from the National Institute for Health and Welfare (THL/1370/5.05.00/2010), Statistics Finland (TK-53-1560-10), and the Social Insurance Institution of Finland (KELA 40/522/2010). All data were pseudonymised before inclusion into the study database.

Data sharing: No additional data are available.

The lead author affirms that the manuscript is an honest, accurate, and transparent account of the study being reported; that no important aspects of the study have been omitted; and that any discrepancies from the study as originally planned (and, if relevant, registered) have been explained.

This is an Open Access article distributed in accordance with the Creative Commons Attribution Non Commercial (CC BY-NC 4.0) license, which permits others to distribute, remix, adapt, build upon this work non-commercially, and license their derivative works on different terms, provided the original work is properly cited and the use is noncommercial. See: http://creativecommons.org/licenses/by-nc/4.0/.

1 Andersen K, Launer LI, Dewey ME, et al, EURODEM Incidence Research Group. Gender differences in the incidence of $A D$ and vascular dementia: The EURODEM Studies. Neurology 1999;53:1992-7. doi:10.1212/WNL.53.9.1992

2 Depypere H, Vierin A, Weyers S, Sieben A. Alzheimer's disease, apolipoprotein $\mathrm{E}$ and hormone replacement therapy. Maturitas 2016:94:98-105. doi:10.1016/i.maturitas.2016.09.009

3 Pike CJ. Sex and the development of Alzheimer's disease. J Neurosci Res 2017;95:671-80. doi:10.1002/jnr.23827
4 Morinaga A, Hirohata M, Ono K, Yamada M. Estrogen has antiamyloidogenic effects on Alzheimer's $\beta$-amyloid fibrils in vitro. Biochem Biophys Res Commun 2007;359:697-702. doi:10.1016/j. bbrc.2007.05.158

5 Yue X, Lu M, Lancaster T, et al. Brain estrogen deficiency accelerates Abeta plaque formation in an Alzheimer's disease animal model. Proc Natl Acad Sci U S A 2005;102:19198-203. doi:10.1073/ pnas. 0505203102

6 Pike CJ, Carroll IC, Rosario ER, Barron AM. Protective actions of sex steroid hormones in Alzheimer's disease. Front Neuroendocrinol 2009;30:239-58. doi:10.1016/j. yfrne.2009.04.015

7 Bove R, Secor E, Chibnik LB, et al. Age at surgical menopause influences cognitive decline and Alzheimer pathology in older women. Neurology 2014;82:222-9. doi:10.1212/ WNL.0000000000000033

8 Tang MX, Jacobs D, Stern Y, et al. Effect of oestrogen during menopause on risk and age at onset of Alzheimer's disease. Lancet 1996;348:429-32. doi:10.1016/S01406736(96)03356-9

9 Waring SC, Rocca WA, Petersen RC, O’Brien PC, Tangalos EG, Kokmen E. Postmenopausal estrogen replacement therapy and risk of AD: a population-based study. Neurology 1999:52:965-70. doi:10.1212/ WNL.52.5.965

10 Zandi PP, Carlson MC, Plassman BL, et al, Cache County Memory Study Investigators. Hormone replacement therapy and incidence of Alzheimer disease in older women: the Cache County Study. JAMA 2002;288:2123-9. doi:10.1001/jama.288.17.2123

11 Shao H, Breitner JCS, Whitmer RA, et al, Cache County Investigators. Hormone therapy and Alzheimer disease dementia: new findings from the Cache County Study. Neurology 2012;79:1846-52. doi:10.1212/WNL.0b013e318271f823

12 Imtiaz B, Taipale H, Tanskanen A, et al. Risk of Alzheimer's disease among users of postmenopausal hormone therapy: A nationwide case-control study. Maturitas 2017;98:7-13. doi:10.1016/j. maturitas.2017.01.002

13 Imtiaz B, Tuppurainen M, Rikkonen T, et al. Postmenopausal hormone therapy and Alzheimer disease: A prospective cohort study. Neurology 2017;88:1062-8. doi:10.1212/ WNL.0000000000003696

14 Shumaker SA, Legault C, Rapp SR, et al, WHIMS Investigators. Estrogen plus progestin and the incidence of dementia and mild cognitive impairment in postmenopausal women: the Women's Health Initiative Memory Study: a randomized controlled trial. JAMA 2003;289:2651-62. doi:10.1001/jama.289.20.2651

15 Shumaker SA, Legault C, Kuller L, et al, Women's Health Initiative Memory Study. Conjugated equine estrogens and incidence of probable dementia and mild cognitive impairment in postmenopausal women: Women's Health Initiative Memory Study. JAMA 2004;291:2947-58. doi:10.1001/jama.291.24.2947

16 Maki PM. Critical window hypothesis of hormone therapy and cognition: a scientific update on clinical studies. Menopause 2013:20:695-709. doi:10.1097| GME.0b013e3182960cf8

17 Harman SM, Vittinghoff E, Brinton EA, et al. Timing and duration of menopausal hormone treatment may affect cardiovascular outcomes. Am J Med 2011;124:199-205. doi:10.1016/j. amjmed.2010.09.021

18 Mikkola TS, Tuomikoski P, Lyytinen H, et al. Estradiol-based postmenopausal hormone therapy and risk of cardiovascular and all-cause mortality. Menopause 2015;22:976-83. doi:10.1097/ GME.0000000000000450

19 Lyytinen H, Pukkala E, Ylikorkala O. Breast cancer risk in postmenopausal women using estradiol-progestogen therapy. Obstet Gynecol 2009;113:65-73. doi:10.1097/AOG.0b013e31818e8cd6

20 Savolainen-Peltonen H, Tuomikoski P, Korhonen P, et al. Cardiac death risk in relation to the age at initiation or the progestin component of hormone therapies. I Clin Endocrinol Metab 2016;101:2794-801. doi:10.1210/ic.2015-4149

21 Mikkola TS, Savolainen-Peltonen H, Tuomikoski P, et al. Lower death risk for vascular dementia than for Alzheimer's disease with postmenopausal hormone therapy users. J Clin Endocrinol Metab 2017:102:870-7.

22 O'Brien J, Jackson JW, Grodstein F, Blacker D, Weuve J. Postmenopausal hormone therapy is not associated with risk of all-cause dementia and Alzheimer's disease. Epidemiol Rev 2014;36:83-103. doi:10.1093/epirev/mxt008

23 Stanczyk FZ, Hapgood JP, Winer S, Mishell DRJr. Progestogens used in postmenopausal hormone therapy: differences in their pharmacological properties, intracellular actions, and clinical effects. Endocr Rev 2013;34:171-208. doi:10.1210/er.2012-1008

24 Pinto-Almazán R, Segura-Uribe JJ, Farfán-García ED, Guerra-Araiza C. Effects of Tibolone on the Central Nervous System: Clinical and Experimental Approaches. Biomed Res Int 2017;2017:8630764. doi:10.1155/2017/8630764 
25 Mucke L, Selkoe DJ. Neurotoxicity of amyloid $\beta$-protein: synaptic and network dysfunction. Cold Spring Harb Perspect Med 2012;2:a006338. doi:10.1101/cshperspect.a006338

26 Zempel H, Mandelkow E. Lost after translation: missorting of Tau protein and consequences for Alzheimer disease. Trends Neurosci 2014;37:721-32. doi:10.1016/j.tins.2014.08.004

27 Jang H, Bae JB, Dardiotis E, et al. Differential effects of completed and incomplete pregnancies on the risk of Alzheimer disease. Neurology 2018;91:e643-51. doi:10.1212/ WNL.0000000000006000

28 Kantarci K, Lowe VJ, Lesnick TG, et al. Early Postmenopausal Transdermal $17 \beta$-Estradiol Therapy and Amyloid- $\beta$ Deposition. J Alzheimers Dis 2016:53:547-56. doi:10.3233/JAD-160258

29 Manson JE, Aragaki AK, Rossouw JE, et al, WHI Investigators Menopausal hormone therapy and long-term all-cause and causespecific mortality: The Women's Health Initiative randomized trials. JAMA 2017;318:927-38. doi:10.1001/jama.2017.11217

30 Tuomikoski P, Salomaa V, Havulinna A, et al. Decreased mortality risk due to first acute coronary syndrome in women with postmenopausal hormone therapy use. Maturitas 2016;94:106-9. doi:10.1016/j. maturitas.2016.09.015

31 Corbo RM, Scacchi R. Apolipoprotein E (APOE) allele distribution in the world. Is APOE*4 a 'thrifty' allele?Ann Hum Genet 1999;63:30110. doi:10.1046/j.1469-1809.1999.6340301.x
32 Ewbank DC. The APOE gene and differences in life expectancy in Europe. J Gerontol A Biol Sci Med Sci 2004;59:16-20. doi:10.1093/ gerona/59.1.B16

33 Topo P, Luoto R, Hemminki E, Uutela A. Declining socioeconomic differences in the use of menopausal and postmenopausal hormone therapy in Finland. Maturitas 1999;32:141-5. doi:10.1016/S03785122(99)00027-4

34 Wilson RS, Segawa E, Boyle PA, Anagnos SE, Hizel LP, Bennett DA. The natural history of cognitive decline in Alzheimer's disease. Psychol Aging 2012;27:1008-17. doi:10.1037/a0029857

35 Lobo A, Launer LJ, Fratiglioni L, et al, Neurologic Diseases in the Elderly Research Group. Prevalence of dementia and major subtypes in Europe: A collaborative study of population-based cohorts. Neurology 2000;54(Suppl 5):S4-9.

36 Dubois B, Feldman HH, Jacova C, et al. Research criteria for the diagnosis of Alzheimer's disease: revising the NINCDS-ADRDA criteria. Lancet Neurol 2007;6:734-46. doi:10.1016/S14744422(07)70178-3

37 Taipale H, Tanskanen A, Koponen M, Tolppanen AM, Tiihonen J, Hartikainen S. Antidementia drug use among community-dwelling individuals with Alzheimer's disease in Finland: a nationwide register-based study. Int Clin Psychopharmacol 2014:29:216-23. doi:10.1097/ YIC.0000000000000032 